\title{
Effectiveness of Physiotherapy Treatment on Clinical Features and Quality of Life of COVID-19 Positive Patients
}

\author{
Vidhi D. Thakkar ${ }^{1}$, Sweety Shah ${ }^{2}$ \\ ${ }^{1}$ Post-Graduate Student, SBB College of Physiotherapy, VS General Hospital, SVP Hospital, Ahmedabad, India. \\ ${ }^{2}$ Ph.D, MPT, SBB College of Physiotherapy, VS General Hospital, SVP Hospital, Ahmedabad, India.
}

Corresponding Author: Vidhi D. Thakkar

\begin{abstract}
Background: World Health Organization has declared corona virus disease (COVID 19) a global pandemic. Fever, dry cough and breathlessness are the main manifestations. In addition, myalgia, muscle weakness, fatigue may render the patients unable to carry out activities of daily living (ADL) which affect patient's QOL. Amongst health workers, physiotherapists are playing an important role in managing and caring novel COVID-19 patients in ICUs and also out there. Clinical features-Pain, Muscle weakness, Fatigue was assessed by following Numeric pain rating scale, Manual muscle testing, Fatigue assessment scale. Functional status and Quality of life of patients, measured by functional independent measurement scale, McGill Quality of life Questionnaire. So, aim is to provide evidence of physiotherapist involvement in managing COVID-19 infected patients.

Materials and Methods: Interventional study, simple random sampling with sample size of sixteen Covid 19 positive patients was done. Both male and female included. Patients who were mentally ill excluded. Oral consent was taken. Subjects were given physiotherapy treatment for 1 week. (Seven days) which include chest physiotherapy, Breathing exercises, early mobilization, Limb physiotherapy, Positioning etc. Clinical features of Covid 19-Pain, Muscle strength, Fatigue level, Functional status and Quality of life was measured before and after intervention. Wilcoxon test used to analyze data.

Results: Data of 16 subjects was analyzed. Result showed that significant decrease in pain, fatigue level and increase in muscle strength, functional status and QOL.

Conclusion: Physiotherapy treatment improves QOL and clinical features of covid 19 patients like pain, muscle strength, fatigue.
\end{abstract}

Key Words: Physiotherapy, Pain, Muscle strength, Fatigue, functional level, QOL.

\section{INTRODUCTION}

World Health Organization has declared corona virus disease (COVID 19) a global pandemic and public health emergency. Fever, dry cough and breathlessness are the main manifestations. In addition, symptoms such as myalgia, muscle weakness, and headache, fatigue may render the patients unable to carry out activities of daily living (ADL) which affect patient's QOL.
Amongst health-care workers, physical-therapists, especially respiratory therapists, are also playing an important role in managing and caring novel COVID-19 patients in ICUs and also out there. They are involved in conservative care, posture correction, mobilization and training of weaning from invasive mechanical ventilator support.

Critically ill patients undergo a significant pain burden during everyday procedures in ICU, such as tracheal tube 
suctioning, turning, positioning, and different line insertion. Muscle weakness can result in complications such as muscle atrophy and contracture in the long term." Fatigue" is an impaired ability to perform a mental or physical task as a result of depleted mental or physical resources. [1] Some of these impairments can be severe and persist for years after critical illness, resulting in poor quality of life (QOL). ${ }^{[2]}$

Identifying the clinical features of the disease would be beneficial and can provide further information which will help to understand the diagnosis and management of Covid 19.

Different signs and symptoms like pain, muscle weakness, fatigue are of great concern in Covid 19 positive patients which affect quality of life of patient and whole body functions.

There are Small numbers of studies on rehabilitation for specific symptoms of Covid 19 patients.

So the Aim is to find out effectiveness of physiotherapy treatment on clinical features of Covid 19 and QOL (Quality of life) of COVID-19 positive patients.

\section{MATERIALS AND METHODS}

An Experimental study was conducted at SVP Hospital of Ahmedabad. A total of 16 subjects, both males and females, aged between 18-70 years who were Covid positive were included in the study by simple random sampling. The subjects who had recent fractures, mentally ill, unconscious were excluded. Information about the purpose and procedure of the study was provided to the participants. Informed consent was taken from them prior to participation. Outcome measures were Pain, Muscle weakness, Fatigue, Functional status, Quality of life assessed by following NPRS (Numeric pain rating scale), MMT (Manual muscle testing), FAS (Fatigue assessment scale), FIM (Functional independent measurement scale), McGill QOL (McGill Quality of life Questionnaire).

\section{Procedure of the study:}

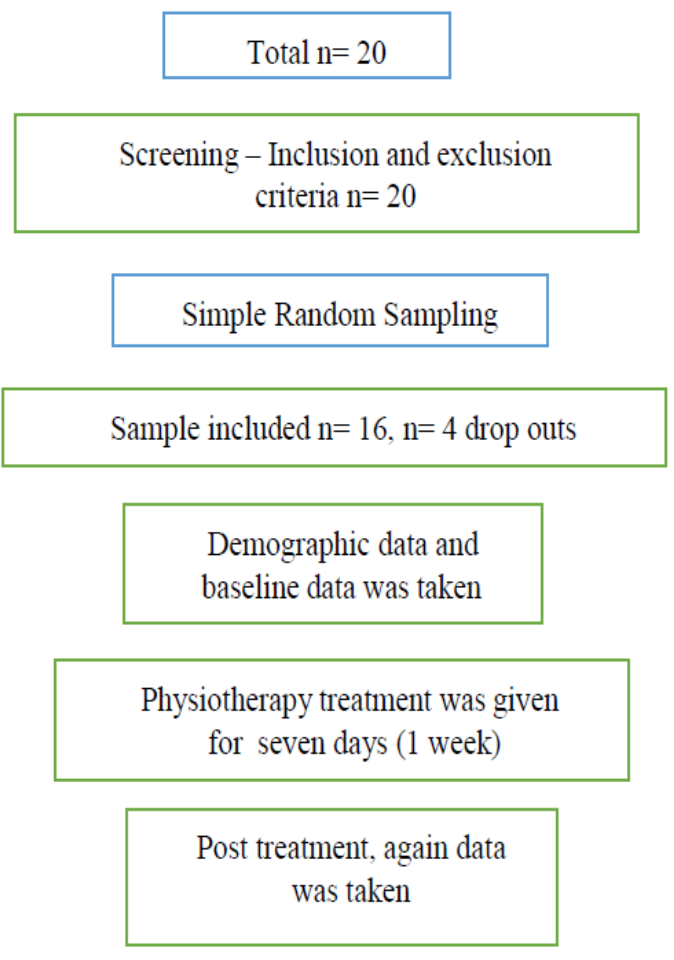

\section{Physiotherapy treatment:}

Physiotherapy treatment was given to the patients two times a day for one week.

Which include chest physiotherapyDeep Breathing exercise, Diaphragmatic breathing exercise, pursed lip breathing, chest expansion exercise, Breathing control.

Limb physiotherapy-Active assisted limb exercises, Active limb exercise

Assistive device-Red rubber balloon and EZ PEP. positions.

Positioning-Prone and side lying

Early mobilization-Prop up sitting, high sitting, standing, marching, walking, Step up, Step down.

\section{A. Pain}

The individual were checked their pain by NPRS (Numeric pain rating scale). NPRS is an 11-point scale from 0-10. In which " $0 "=$ No pain and " $10 "=$ the most intense pain imaginable. Patients were verbally asked to select value which was most in line with the intensity of pain that they have experienced. 


\section{B. Muscle weakness}

The individual were checked their muscle weakness by Oxford muscle grading (Manual muscle testing).This method involves testing key muscles from the upper and lower extremities against the examiner's resistance and grading the patient's strength on a 0 to 5 scale accordingly. Grades are as follows-0 -No muscle movement ,1-Flicker of contraction ,2-Active movement with gravity eliminated through full range,3-Active movement against gravity through full range,4-Active movement against gravity with some resistance,5-Active movement against gravity with strong resistance

\section{Fatigue}

The individual were checked their Fatigue by fatigue assessment scale (FAS).The FAS is a 10-item scale. Each item of the FAS was answered by using a five-point Scale ranging from 1 ("never") to 5 ("always"). 1= Never, 2= Sometimes, $3=$ Regularly,4= Often, $5=$ Always. Total scores can range from 10, indicating the lowest level of fatigue, to 50, denoting the highest.

\section{Functional Status of patient}

The individual were checked their Functional status by FIM (Functional Independence Measure) Scale. Functional Independence Measure (FIM) is an 18-item measurement tool that explores an individual's physical, psychological and social function. The tool is used to assess a patient's level of disability as well as a change in patient status in response to rehabilitation or medical intervention. The grading categories range from "total assistance with helper $=1$ " to "complete independence with no helper=7".

\section{E. Quality of life}

The individual were checked their Quality of life by McGill QUALITY OF LIFE QUESTIONNAIRE. Circle the number between 0 to 10 which was most true for the patient. No right or wrong answers.

\section{STATISTICAL ANALYSIS}

Statistical analysis was done by using SPSS version 16. Level of significance was kept at 5\%. Descriptive statistics for all outcome measures were expressed as mean, standard deviations. Data was considered statistically significant with $\mathrm{p}$ value $<0.05$ and highly significant with $\mathrm{p}$ value $<0.01$. Nonparametric Wilcoxon test used to analyze data.

\section{RESULTS}

Data of 16 subjects was analyzed. Subject showed that there was significant decrease in pain, fatigue level and increase in muscle strength, functional status and QOL following 1 week of physiotherapy treatment. Difference between 1 st and 7 th day ranges (mean $\& S D$ ) shows in table. 1

Table: 1-pre \& post MEAN \pm SD for all outcome measures.
\begin{tabular}{|l|l|l|l|l|l|}
\hline SR.NO & VARIABLE & PRE MEAN \pm SD & POST MEAN \pm SD & P VALUE & SIGNIFICANCE \\
\hline 1 & Pain & $4.6250 \pm 2.02896$ & $1.0000 \pm 1.63299$ & 0.000 & Highly significant \\
\hline 2 & Muscle Weakness & $3.5625 \pm 0.62915$ & $4.5625 \pm 0.5123$ & 0.000 & Highly significant \\
\hline 3 & Fatigue & $20.5625 \pm 7.5185$ & $17.3750 \pm 10.42353$ & 0.007 & Significant \\
\hline 4 & Functional Status & $85.500 \pm 22.559$ & $108.5 \pm 18.3484$ & 0.000 & Highly significant \\
\hline 5 & Quality Of Life & $69.375 \pm 13.5640$ & $82.4375 \pm 10.60190$ & 0.000 & Highly significant \\
\hline
\end{tabular}

The mean baseline value for pain was 4.6250 \pm 2.02896 . After intervention the mean Value for pain 1.0000 \pm 1.63299 (table $1 \&$ graph 1). The mean value for Pretreatment and post-treatment Pain was 4.6250 and 1.0000 respectively and the standard deviation was 2.02896 and 1.63299 respectively. As $\mathrm{p}=0.000$, the result was highly statistically significant.

The mean baseline value for muscle strength was 3.5625 \pm 0.62915 .After intervention the mean value for muscle strength was $4.5625 \pm 0.51235$ (table 1 \& graph 1). The mean value for Pre- treatment and Post-treatment muscle strength was 
3.5625 and 4.5625 respectively and the standard deviation was 0.62915 and 0.51235 respectively. As $\mathrm{p}=0.000$, the result was highly statistically significant.

The mean baseline value for fatigue was 20.5625 \pm 7.5185 . After intervention the mean value for fatigue was $17.3750 \pm$ 10.42353 (table $1 \&$ graph 1). The mean value for Pre-treatment and Post- treatment fatigue was 20.5625 and 17.3750 respectively and the standard deviation was 7.5185 and 10.42353 respectively. As $\mathrm{p}=0.007$, the result was statistically significant.

The mean baseline value for functional level was $85.500 \pm 22.5595$.After intervention the mean value for functional level was $108.5 \pm 18.3484$ (table $1 \&$ graph $1)$. The mean value for Pre- treatment and Post-treatment functional level was 85.500 and 108.5 respectively and the standard deviation is 22.5595 and 18.3484 respectively. As $\mathrm{p}=0.000$, the result was highly statistically significant.

The mean baseline value for QOL was 69.375 \pm 13.5640 .After intervention the mean value for functional level was 82.4375 \pm 10.60190 (table 1 \& graph 1 ). The mean value for Pre-treatment and Post-treatment functional level was 69.375 and 82.4375 respectively and the standard deviation was 13.5640 and 10.60190 respectively. As $\mathrm{p}=0.000$, the result is highly statistically significant.

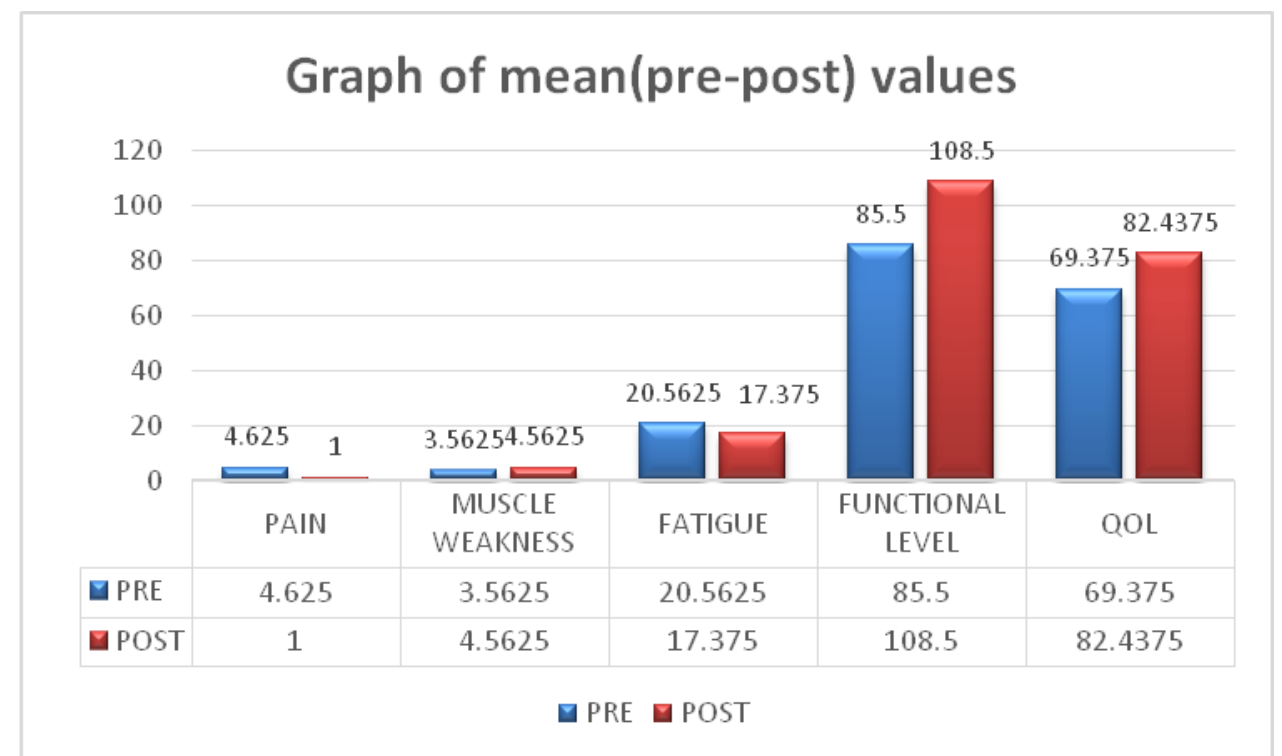

Graph-1: pre and post intervention values (mean \pm SD) for pain, fatigue, muscle weakness, functional level and QOL

\section{DISCUSSION}

Covid 19 is considered as world pandemic disease in which patient suffers from musculoskeletal, respiratory impairments along with psychosocial issues. Bed ridden patients develop secondary complications following primary disease. So Early mobilization is very important to prevent secondary complications.

Limb physiotherapy associated with chest physiotherapy improves lung function as Covid 19 affects the lung most. This ultimately improves functional level of patient and Quality of life.
P.Thomas et al. conducted study on physiotherapy management for Covid 19 in acute hospital setting and concluded that physiotherapists are responsible for early mobilization of patient including active, active assisted, passive ROM exercise to improve joint integrity, bed mobility. Muscle strength, sitting, standing, sit to stand and walking. ${ }^{[3]}$

Radha Korupolu et al. conducted study on Rehabilitation of critically Ill COVID-19 survivors and concluded that Rehabilitation of critically ill COVID-19 survivors is important to reduce long-term complications. A comprehensive 
rehabilitation program, with a multidisciplinary approach, is needed to reduce such (ICUAW, dysphagia, functional decline, psychological problems, cognitive impairment, and reduced QOL) complications. ${ }^{[4]}$

Muscle strength of body has direct correlation to functional level of status and QOL of patient. If weakness decreases gradually, patient can perform their ADL activities by their own. They don't need assistance for their daily living activities. Ultimately QOL of patient will be increased.

Fatigue limits the patient's active participation. Fatigue can be mental or physical. By the relaxation techniques and energy conservation techniques it decreases gradually.

Mandeep Kumar Jangra et al. conducted study on Significance of physiotherapy in "SARS-CoV- 2/COVID19: An Epidemic" and concluded that it is important to anticipate early rehabilitation to prevent or limit the ICU- acquired weakness. Physiotherapists provide exercises, early mobilization, and rehabilitation strategy in patients with SARS-CoV-2/COVID-19 to make them functionally independent at the time of discharge from hospital. ${ }^{[5]}$

Early rehabilitation in critical care is very important which help to early mobilization of patients. Patients can manage to sit upright after prolong bed ridden positions which improves ventilation in the lungs. Gradually patient can stand and walk independently which leads to step and step down even without breathlessness which is most seen complaint in Covid patients.

Positioning in bed in every 2 hourly, helps to prevent secondary complications of bed ridden patients. Proning helps to improve oxygenation of the lungs. Assistive device like Red rubber balloon and EZ PEP helps to improve forceful expiration which in turn helps to clearance of secretions.

\section{CONCLUSION}

From the results of the study, Physiotherapy treatment is beneficial to improve clinical features of Covid positive patients and improves QOL by strengthening body as well as respiratory system.

\section{Limitation \& Future Scope of the Study}

The Subjects for study was taken only from one hospital of Ahmedabad. The future study can be done on role of physiotherapy in post Covid (post Covid rehab) complications after discharged from hospital.

\section{Clinical Implications}

An individually tailored Physiotherapy regimen helps COVID 19 patients towards a successful recovery and improves their quality of life.

\section{ACKNOWLEDGEMENT}

Special Thanks to my guide for encouraging and supporting me always throughout my study.

I am thankful to all the subjects who participated in my study to make this study successful.

I want to thank my parents and colleagues for their motivation and help throughout my study.

\section{Conflict of Interest: None}

\section{Source of Funding: None}

\section{Ethical Approval: Approved}

\section{REFERENCES}

1. Kemp, Harriet I., Eve Corner, and Lesley A. Colvin. "Chronic pain following COVID19: implications for rehabilitation." BJA: British Journal of Anesthesia (2020).

2. Michie, Susan, Robert West, and Nigel Harvey. "The concept of "fatigue" in tackling Covid- 19." BMJ 371 (2020).

3. Thomas, P. "Physiotherapy management for COVID-19 in the acute hospital setting." ПINEYM $\Omega$ N 33.1 (2020): 35. 
Vidhi D. Thakkar et.al. Effectiveness of physiotherapy treatment on clinical features and quality of life of Covid19 positive patients.

4. Korupolu, Radha, et al. "Rehabilitation of critically ill COVID-19 survivors." The Journal of the International Society of Physical and Rehabilitation Medicine 3.2 (2020): 45 .

5. Jangra, Mandeep Kumar, and Akanksha Saxena. "Significance of physiotherapy in "SARS- CoV-2/COVID-19: An Epidemic"." Annals of Thoracic Medicine 15.3 (2020): 179.

6. Kachpile, Sheral T., et al. "Physiotherapy interventions in COVID-19 patient with multiple comorbidities: a case report." Int J Health Sci Res. 2020; 10 (10); 96-101.

7. Özkeskin, Mehmet, Nuray Elibol, and Serkan Bakırhan. "Risk of COVID-19 Disease in the Elderly Population and Physiotherapy." Physiotherapy (2020).
8. Geete, Dipti Baban, Jaimala Vijay Shetye, and Anil Manohar Sathe. "Physiotherapy management of geriatric COVID-19 patients in an intensive care unit of a government tertiary care hospital: A case series." World Journal of Advanced Research and Reviews 8.3 (2020): 007- 013

9. Abdullahi, Auwal, et al. "Neurological and musculoskeletal features of COVID-19: a systematic review and meta-analysis." Frontiers in neurology 11 (2020): 687.

How to cite this article: Thakkar VD, Shah S. Effectiveness of physiotherapy treatment on clinical features and quality of life of Covid-19 positive patients. Int J Health Sci Res. 2021; 11(5): 299-304. DOI: https://doi.org/10.52403/ ijhsr.20210547 